\title{
Optimal investment and consumption decision of a family with life insurance
}

\author{
Minsuk Kwak ${ }^{\mathrm{a}, *}$, Yong Hyun Shin ${ }^{\mathrm{b}}, \mathrm{U}$ Jin Choi ${ }^{\mathrm{a}}$ \\ a Department of Mathematical Sciences, Korea Advanced Institute of Science and Technology (KAIST), Daejeon, 305701, Republic of Korea \\ ${ }^{\mathrm{b}}$ Department of Mathematics, Hannam University, Daejeon, 306791, Republic of Korea
}

\section{A R T I C L E I N F O}

\section{Article history:}

Received September 2009

Received in revised form

September 2010

Accepted 25 October 2010

\section{IME Subject and Insurance Branch Category:}

IE13

IB11

JEL classification:

D91

E21

G11

G22

Keywords:

Life insurance

Optimal investment/consumption

Labor income

Utility maximization

Martingale method

\begin{abstract}
A B S T R A C T
We study an optimal portfolio and consumption choice problem of a family that combines life insurance for parents who receive deterministic labor income until the fixed time $T$. We consider utility functions of parents and children separately and assume that parents have an uncertain lifetime. If parents die before time $T$, children have no labor income and they choose the optimal consumption and portfolio with remaining wealth and life insurance benefit. The object of the family is to maximize the weighted average of utility of parents and that of children. We obtain analytic solutions for the value function and the optimal policies, and then analyze how the changes of the weight of the parents' utility function and other factors affect the optimal policies.
\end{abstract}

(c) 2010 Elsevier B.V. All rights reserved.

\section{Introduction}

We investigate an optimal investment and consumption decision problem of the family with life insurance for parents. We assume that parents have an uncertain lifetime and while they are alive, they receive deterministic labor income until the fixed time $T>0$. If parents die before time $T$, children have no labor income until time $T$ and they choose their optimal policies with remaining wealth and life insurance benefit. We consider utility functions of parents and children separately. The object of the family is to maximize the weighted average of utility of parents and that of children. It is assumed that the utility functions of both parents and children belong to HARA utility class. Using HARA utility we impose the condition that instantaneous consumption rate should be above a given lower bound. Using the martingale method, analytic solutions for the value function and the optimal policies are derived. We analyze how the changes of the weight of the parents' utility function and other factors, such as the

\footnotetext{
* Corresponding author. Tel.: +82423502766.

E-mail addresses: minsuk.kwak@gmail.com (M. Kwak),yhshin@hnu.kr (Y.H. Shin), ujinchoi@kaist.ac.kr (U.J. Choi).
}

family's current wealth level and the fair discounted value of future labor income, affect the optimal policies and also illustrate some numerical examples.

Since the 1960s, there have been much research on the analysis of the demand for life insurance. Yaari (1965) considered a consumption and life insurance premium choice problem and showed that if an individual has an uncertain lifetime and no bequest motive, it is optimal to annuitize all of savings. Hakansson (1969) examined life-cycle pattern of consumption and saving in a discrete time environment and found conditions under which zero insurance premium is optimal. Fischer (1973) also examined the optimal life insurance purchase by using the dynamic programming methods and obtained the formula of present value of future income which is different from the formula under a certain lifetime. Richard (1975) combined life insurance with the well-known continuous-time model of Merton (1969, 1971) for the optimal consumption and investment. Richard (1975) assumed that the individual's lifetime is bounded and imposed a condition that the individual cannot purchase life insurance at the end of his lifetime. Also Campbell (1980), Lewis (1989), Hurd (1989) and Babbel and Ohtsuka (1989) examined the life insurance problems from different perspectives and gave us numerous insights into the demand for life insurance. 
Recently, Moore and Young (2006) studied the optimal consumption, investment, and insurance strategies for an individual. Pliska and Ye (2007) studied the optimal life insurance and consumption rules for a wage earner whose lifetime is random and Nielsen and Steffensen (2008) investigated optimal investment, and life insurance strategies under minimum and maximum constraints. Ye $(2006,2007)$ considered an optimal life insurance, consumption and portfolio choice problem with an uncertain lifetime and solved the problem by using the martingale method which we use to solve our problem. Huang et al. (2008), and Huang and Milevsky (2008) investigated an optimal life insurance, consumption and portfolio choice problem with a stochastic income process. They focused on the effect of correlation between the dynamics of financial capital and human capital on the optimal policies. They used a similarity reduction technique to find the numerical solution efficiently and found that the life insurance hedges human capital and is insensitive to the risk aversion.

In addition to Merton (1969, 1971), many studies about continuous-time optimal investment and consumption choice problems have been carried out by Karatzas et al. (1986), Pliska (1986), Karatzas et al. (1987), and Cox and Huang (1989) and so on. Recently, portfolio selection problems under a preference change are investigated by Choi and Koo (2005), Stabile (2006), and Kwak et al. (2009).

The rest of this paper proceeds as follows. In Section 2, we introduce our financial market and insurance market setup and derive the budget constraint of the family. We present the optimization problem of the family in Section 3. We derive the analytic solution for the value function of the family's optimization problem and give the optimal policies in explicit forms. In Section 4 we analyze the effects of varying parameters on the optimal solutions with numerical examples, and Section 5 concludes. All detailed proofs in this paper are given in the appendices.

\section{The economy}

We consider an optimization problem of one family until the fixed parents' retirement time $T>0$ in the financial market and the insurance market. In the financial market, it is assumed that there are one risk-free asset and one risky asset which evolve according to the ordinary differential equation (ODE) and to the stochastic differential equation (SDE)

$\mathrm{d} S_{t}^{0}=r S_{t}^{0} \mathrm{~d} t$ and $\mathrm{d} S_{t}^{1}=\mu S_{t}^{1} \mathrm{~d} t+\sigma S_{t}^{1} \mathrm{~d} W_{t}$,

respectively, where $r, \mu$ and $\sigma$ are constants, $W_{t}$ is a standard Brownian motion on a complete probability space $(\Omega, \mathcal{F}, \mathbb{P})$, and $\left\{\mathcal{F}_{t}\right\}_{t=0}^{T}$ is the $\mathbb{P}$-augmentation of the natural filtration generated by $W_{t}{ }^{1}$

Let $c_{p}(t)$ be the consumption rate of parents at time $t, c_{c}(t)$ be the consumption rate of children at time $t$ and $\pi(t)$ be the amount invested in the risky asset $S_{t}^{1}$ at time $t$. It is assumed that the consumption rate processes $c_{p}(t)$ and $c_{c}(t)$ are nonnegative, $\mathcal{F}_{t}$-progressively measurable, satisfying, respectively,

$\int_{0}^{T} c_{p}(t) \mathrm{d} t<\infty, \quad$ almost surely (a.s.) and

$\int_{0}^{T} c_{c}(t) \mathrm{d} t<\infty, \quad$ a.s.

and the portfolio process $\pi(t)$ is $\mathcal{F}_{t}$-adapted, satisfying

$$
\int_{0}^{T} \pi(t)^{2} \mathrm{~d} t<\infty, \quad \text { a.s. }
$$

\footnotetext{
${ }^{1}$ For more details, refer Section 1.1 of Karatzas and Shreve (1998).
}

Let $\tau$ be the death time of parents who have the deterministic labor income $w_{t}$ until time $T$. $^{2}$ It is assumed that the lifetime $\tau$ is independent of the filtration $\left\{\mathcal{F}_{t}\right\}$.

In the insurance market, let $\lambda_{y+t}$ be an instantaneous force of mortality curve, where $y$ is the age of the breadwinner at an initial time of the model. Then the conditional probability of survival, from age $y$ to age $y+t$, under the law of mortality defined by $\lambda_{y+t}$, can be computed by

${ }_{t} p_{y} \triangleq \mathrm{e}^{-\int_{0}^{t} \lambda_{y+s} \mathrm{~d} s}$.

It is assumed that if the family's insurance premium rate at time $t$ is $I(t)$, then they receive lump sum payment $I(\tau) / \lambda_{y+\tau}$ at the parents' death time $\tau$. ${ }^{3}$ Let $X_{t}$ be the family's wealth at time $t$ until a random time $\tau_{m} \triangleq \min (\tau, T)$, then the total legacy $M(t)$, when the parents die at time $t$ with a wealth $X_{t}$, is

$M(t) \triangleq X_{t}+\frac{I(t)}{\lambda_{y+t}}$,

where the family's wealth dynamics $X_{t}$ satisfies the following SDE: $\mathrm{d} X_{t}=\left[r X_{t}+(\mu-r) \pi(t)-c_{p}(t)-c_{c}(t)-I(t)+w_{t}\right] \mathrm{d} t$

$$
+\sigma \pi(t) \mathrm{d} W_{t}, \quad \text { for } 0 \leq t<\tau_{m} \text {. }
$$

Now let us define the market-price-of-risk, the discount process, the exponential martingale process, and the pricing kernel process (or state-price-density process), respectively, by

$\theta \triangleq \frac{\mu-r}{\sigma}, \quad \zeta_{t} \triangleq \mathrm{e}^{-\int_{0}^{t}\left(\lambda_{y+s}+r\right) \mathrm{d} s}$,

$Z_{t} \triangleq \mathrm{e}^{-\theta W_{t}-\frac{1}{2} \theta^{2} t}$ and $H_{t} \triangleq \zeta_{t} Z_{t}$.

The equivalent martingale measure is defined by $\widetilde{\mathbb{P}}(A) \triangleq \mathbb{E}\left[Z_{T} \mathbf{1}_{A}\right]$, for all $A \in \mathcal{F}_{T}$ and the Girsanov theorem implies that $\mathcal{W}_{t} \triangleq$ $W_{t}+\theta t, 0 \leq t \leq T$, is also a standard Brownian motion under the new measure $\widetilde{\mathbb{P}}$. So the wealth dynamics (2.2) until time $\tau_{m}$ can be rewritten as

$$
\begin{aligned}
\mathrm{d} X_{t}= & {\left[r X_{t}-c_{p}(t)-c_{c}(t)-I(t)+w_{t}\right] \mathrm{d} t+\sigma \pi(t) \mathrm{d} \tilde{W}_{t} } \\
= & {\left[\left(r+\lambda_{y+t}\right) X_{t}-c_{p}(t)-c_{c}(t)-\lambda_{y+t} M(t)+w_{t}\right] \mathrm{d} t } \\
& +\sigma \pi(t) \mathrm{d} \tilde{W}_{t}, \quad \text { for } 0 \leq t<\tau_{m} .
\end{aligned}
$$

For $0 \leq t<u$, applying Itô's formula to $\zeta_{t} X_{t}$, we obtain

$$
\begin{gathered}
\zeta_{u} X_{u}+\int_{t}^{u} \zeta_{s}\left(c_{p}(s)+c_{c}(s)+\lambda_{y+s} M(s)\right) \mathrm{d} s \\
=\zeta_{t} X_{t}+\int_{t}^{u} \zeta_{s} w_{s} \mathrm{~d} s+\int_{t}^{u} \zeta_{s} \sigma \pi(s) \mathrm{d} \tilde{W}_{s} .
\end{gathered}
$$

If we define

$b_{u} \triangleq \int_{u}^{T} w_{s} \frac{\zeta_{s}}{\zeta_{u}} \mathrm{~d} s, 4$

and add $\zeta_{u} b_{u}$ on both sides of Eq. (2.3), then we have

$$
\begin{gathered}
\zeta_{u}\left(X_{u}+b_{u}\right)+\int_{t}^{u} \zeta_{s}\left(c_{p}(s)+c_{c}(s)+\lambda_{y+s} M(s)\right) \mathrm{d} s \\
=\zeta_{t}\left(X_{t}+b_{t}\right)+\int_{t}^{u} \zeta_{s} \sigma \pi(s) \mathrm{d} \tilde{W}_{s} .
\end{gathered}
$$

Taking the conditional expectation $\widetilde{\mathbb{E}}_{t}[\cdot]=\widetilde{\mathbb{E}}\left[\cdot \mid \mathcal{F}_{t}\right]$ on both sides of Eq. (2.4) and using Bayes rule, we derive the following budget

\footnotetext{
2 In this paper, it is assumed that parents represent children's father or mother with labor income. It is also assumed that children have no labor income.

3 We focus on the case with zero premium loading. The premium loading can be incorporated into our model by introducing a continuous function $\eta:[0, T] \rightarrow \mathbb{R}^{+}$ which satisfies $\eta(t) \geq \lambda_{y+t}$ and replacing the insurance benefit $\frac{I(\tau)}{\lambda_{y+\tau}}$ with $\frac{I(\tau)}{\eta(\tau)}$.

${ }^{4} b_{t}$ is the fair discounted value of the parents' future labor income from $t$ to $\tau_{m}$.
} 


\section{دريافت فورى ـ ـ متن كامل مقاله}

\section{ISIArticles}

مرجع مقالات تخصصى ايران

ل امكان دانلود نسخه تمام متن مقالات انكليسى ل امكان دانلود نسخه ترجمه شده مقالات ل يذيرش سفارش ترجمه تخصصى $\checkmark$ ل امكان جستجو در آرشيو جامعى از صدها موضوع و هزاران مقاله ل امكان دانلود رايكان r صفحه اول هر مقاله

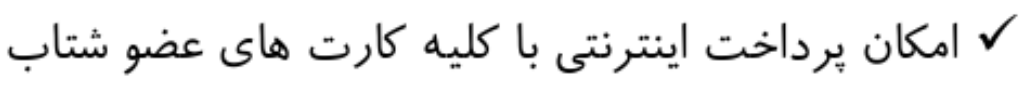
ل دانلود فورى مقاله پِ از برداخت آنلاين

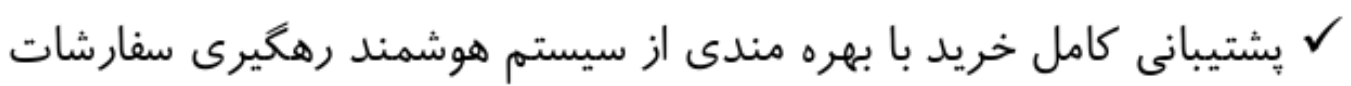

